\title{
GAIA Level 2 Vasa Previa
}

National Cancer Institute

\section{Source}

National Cancer Institute. GAlA Level 2 Vasa Previa. NCI Thesaurus. Code C128747.

GAIA Level 2 Vasa Previa is defined by four criteria: first, vaginal bleeding during the second or third trimester at the time of ruptured amniotic membranes; second, fetal heart rate chang es ultimately resulting in sinusoidal rhythm/terminal bradycardia; third, delivery of one of the following: a) a pale, anemic infant; OR b) a recent stillbirth; OR c) a neonatal death; fourth, post-delivery examination of the placental specimen that demonstrates unsupported fetal vessels within the membranes. 